\title{
A Publicly Available, Annotated Data Set for Naturalistic Driving Study and Computer Vision Algorithm Development
}

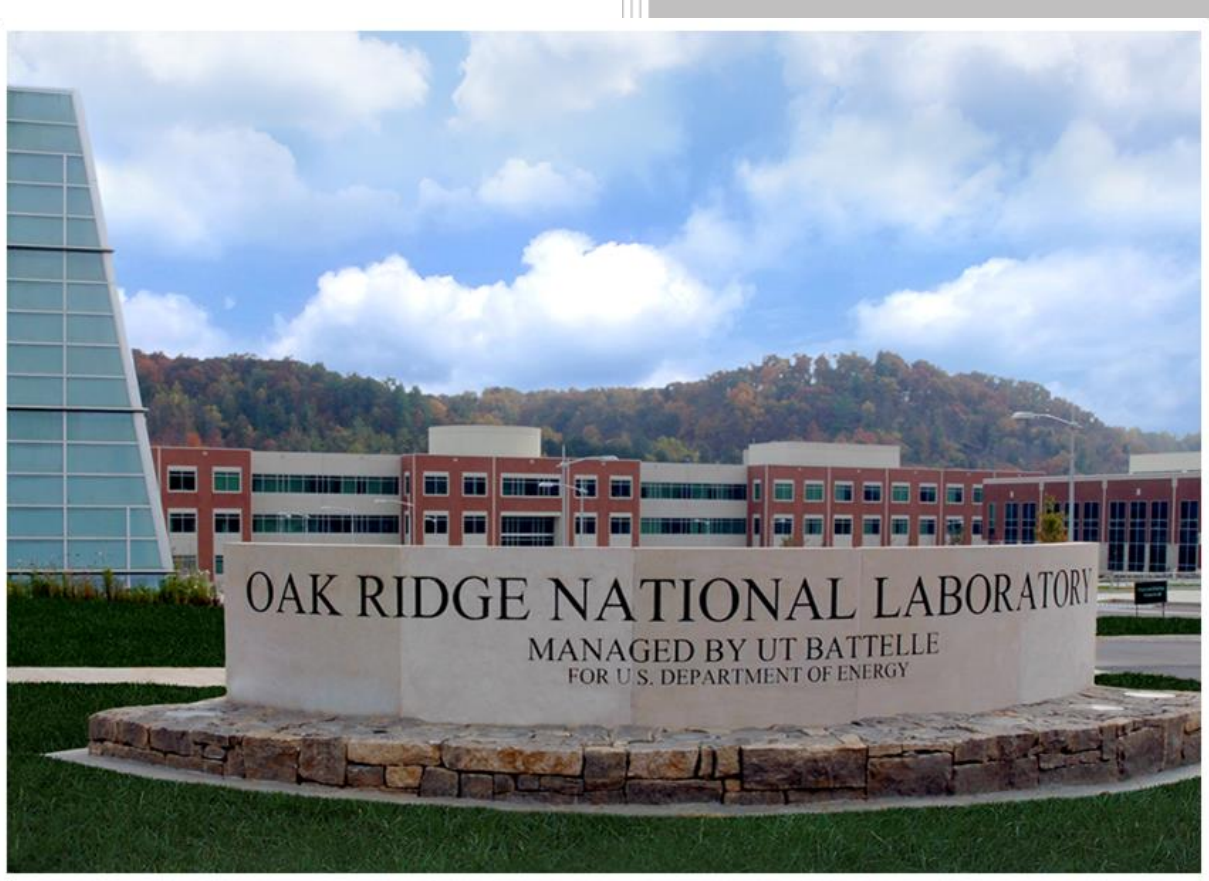

Regina Ferrell Deniz Aykac Tom Karnowski Nisha Srinivas

January 2021 


\title{
DOCUMENT AVAILABILITY
}

Reports produced after January 1, 1996, are generally available free via US Department of Energy (DOE) SciTech Connect.

Website www.osti.gov

Reports produced before January 1, 1996, may be purchased by members of the public from the following source:

\author{
National Technical Information Service \\ 5285 Port Royal Road \\ Springfield, VA 22161 \\ Telephone 703-605-6000 (1-800-553-6847) \\ TDD 703-487-4639 \\ Fax 703-605-6900 \\ E-mail info@ntis.gov \\ Website http://classic.ntis.gov/
}

Reports are available to DOE employees, DOE contractors, Energy Technology Data Exchange representatives, and International Nuclear Information System representatives from the following source:

Office of Scientific and Technical Information

PO Box 62

Oak Ridge, TN 37831

Telephone 865-576-8401

Fax 865-576-5728

E-mail reports@osti.gov

Website http://www.osti.gov/contact.html

This report was prepared as an account of work sponsored by an agency of the United States Government. Neither the United States Government nor any agency thereof, nor any of their employees, makes any warranty, express or implied, or assumes any legal liability or responsibility for the accuracy, completeness, or usefulness of any information, apparatus, product, or process disclosed, or represents that its use would not infringe privately owned rights. Reference herein to any specific commercial product, process, or service by trade name, trademark, manufacturer, or otherwise, does not necessarily constitute or imply its endorsement, recommendation, or favoring by the United States Government or any agency thereof. The views and opinions of authors expressed herein do not necessarily state or reflect those of the United States Government or any agency thereof. 


\section{OBJECTIVE OF COLLECTION}

Oak Ridge National Laboratory developed and implemented a data collection effort to create a dataset for use in evaluating and testing algorithms for analyzing driver behavior under controlled settings for support of the Federal Highway Administration's Exploratory Advanced Research Program. This collection is called the ORNL Naturalistic Driving Study Sample (ONDSS). The dataset is designed to emulate aspects of the Second Strategic Highway Research Project (SHRP2), which contained a massive naturalistic driving study (NDS) with over 3000 drivers between 2010 and 2013 using their personal vehicles, with over 4300 person-years of data collected [HANKEY]. However, due to privacy constraints the video data can only be obtained under strict constraints, and data from the face camera is not available outside secure enclaves at Virginia Tech Transportation Institute in Blacksburg, VA and Turner-Fairbank Research Center in McLean, VA.

The data was collected under Institutional Review Board approval with the understanding that the data will be published and freely available to interested parties. Several data collection goals were accomplished:

1. To create a controlled set of video data where driver head pose was measured by sensors during a set of prescribed motions.

2. To create a set of video data with drivers attempting to convey a particular set of emotions.

3. To create a set of videos with annotated and prescribed usage of a cell phone while simulating driving.

4. To create sets of videos where drivers perform secondary actions that are unrelated to the task of driving and could be indicative of a certain level of distraction. Examples include eating or drinking, dancing, and adjusting various controls and components of the car unrelated to driving such as rolling down the window, talking on the phone, etc.

5. To create a set of videos with the participant using a particular hand or hand combination to drive.

6. To create a set of videos with the participant removing or putting on his/her seat belt.

These activities were grouped into the overall video categories shown in Table 1. All activities were performed while participants were asked to pretend to drive.

Table 1. Explanation of sets of videos generated

\begin{tabular}{llll}
\hline \multicolumn{1}{c}{ Video Category } & \multicolumn{1}{c}{ Description } & \multicolumn{1}{c}{ Special Notes } & Annotation State \\
\hline HeadPose & $\begin{array}{l}\text { Participant turns head to directly } \\
\text { face designated places in the car } \\
\text { relevant to various driving } \\
\text { maneuvers. }\end{array}$ & $\begin{array}{l}\text { Has accelerometer head pose } \\
\text { data; attempts sequence five times }\end{array}$ & Complete \\
Check & $\begin{array}{l}\text { Participant looks at designated } \\
\text { places (same descriptions as in } \\
\text { HeadPose but the participant is } \\
\text { not required to turn the head to } \\
\text { face those locations) in the car } \\
\text { relevant to various driving } \\
\text { maneuvers. }\end{array}$ & $\begin{array}{l}\text { Has accelerometer head pose } \\
\text { data; attempts sequence five times }\end{array}$ & \\
& & & \\
& & & \\
& & & \\
\end{tabular}


Table 1. Explanation of sets of videos generated (continued)

\begin{tabular}{|c|c|c|c|}
\hline Video Category & Description & Special Notes & Annotation State \\
\hline ActionsShorter & $\begin{array}{l}\text { Participant engages in a number } \\
\text { of nondriving activities while } \\
\text { pretending to drive including } \\
\text { singing, blinking, yawning, } \\
\text { grooming, etc. }\end{array}$ & Attempts sequence three times & Complete \\
\hline GlassAndCell & $\begin{array}{l}\text { Participant simulates using a cell } \\
\text { phone in various modes including } \\
\text { talking, texting, and web surfing. }\end{array}$ & Attempts sequence three times & Complete \\
\hline Driving & $\begin{array}{l}\text { Participant actually drives a short } \\
\text { route. }\end{array}$ & Has accelerometer head pose data & None \\
\hline Conversations & $\begin{array}{l}\text { Participant engages in } \\
\text { conversation with people inside } \\
\text { and outside the vehicle }\end{array}$ & Attempts sequence three times & Complete \\
\hline Eating & Participant picks up food and eats & Attempts sequence three times & Complete \\
\hline Expressions & $\begin{array}{l}\text { Participant simulates various } \\
\text { emotional responses including } \\
\text { anger, contempt, disgust, fear, } \\
\text { happy, sadness, and surprise }\end{array}$ & Attempts sequence five times & Complete \\
\hline Hands & $\begin{array}{l}\text { Participant moves through a } \\
\text { sequence of combinations of } \\
\text { hands on steering wheel }\end{array}$ & Attempts sequence three times & Complete \\
\hline HighlyEngaged & $\begin{array}{l}\text { Participant rifles through a bag of } \\
\text { items in passenger seat while } \\
\text { pretending to drive }\end{array}$ & Attempts sequence three times & Complete \\
\hline Seatbelt & $\begin{array}{l}\text { Participant removes and rebuckles } \\
\text { seatbelt }\end{array}$ & Attempts sequence three times & Complete \\
\hline VisorAndWindow & $\begin{array}{l}\text { Participant follows sequence of } \\
\text { actions involving the visor and } \\
\text { rolling down the window }\end{array}$ & Attempts sequence three times & Complete \\
\hline
\end{tabular}

\section{EQUIPMENT AND SOFTWARE}

All testing was done in the same 2004 Honda Civic. This vehicle was instrumented with a loaned set of camera and Data Acquisition System (DAS) used in the Naturalistic Driving studies performed by Virginia Tech Transportation Institute (VTTI) [INSIGHT]. The rear camera of the system was not mounted in the rear window as in the SHRP-2 and Head Pose Validation studies; instead, it was used to help monitor stages of the test. Figure 1 shows the mounting of the unit housing the front, face, and hands camera. This unit produces a single output video, as shown in Figure 2, which is a combination view from all the cameras. The separate views from each camera were extracted and rotated as needed and saved for the data set. In addition, another annotated view was generated combining the camera views with the expanded hand view and the orientation flipped as first performed by SRI International in their "the SRIDCODE method of dual hand-face tracking." An example of an annotated video view can be seen in Figure 3.

In addition to the VTTI camera suite, a small GoPro camera was mounted on the dash directly in front of the driver's seat to acquire concurrent, higher resolution video of the same events. For three of the experiments-HeadPose, Check, and Driving - the driver wore a headpiece mounted with a VectorNav 
VN-100 sensor [VNAV] that rested on the top of the head. This sensor was used to measure the yaw, pitch, and roll pose angles of the driver's head. This sensor was tared for a zero angle corresponding to the driver looking directly at the DAS camera mounted below the rearview mirror. An additional VectorNav VN-100 sensor was mounted in the center of the top of the rear seat to make corrections for measurements when both the car and the driver are in motion. These sensors were found to exhibit considerable drift over time. The data is available in its original form as well as a version where the sensor data was corrected for this drift. This data provides information on the changes in the yaw, pitch and roll of the head during the actions scripted for head pose, check and the short driving segment.

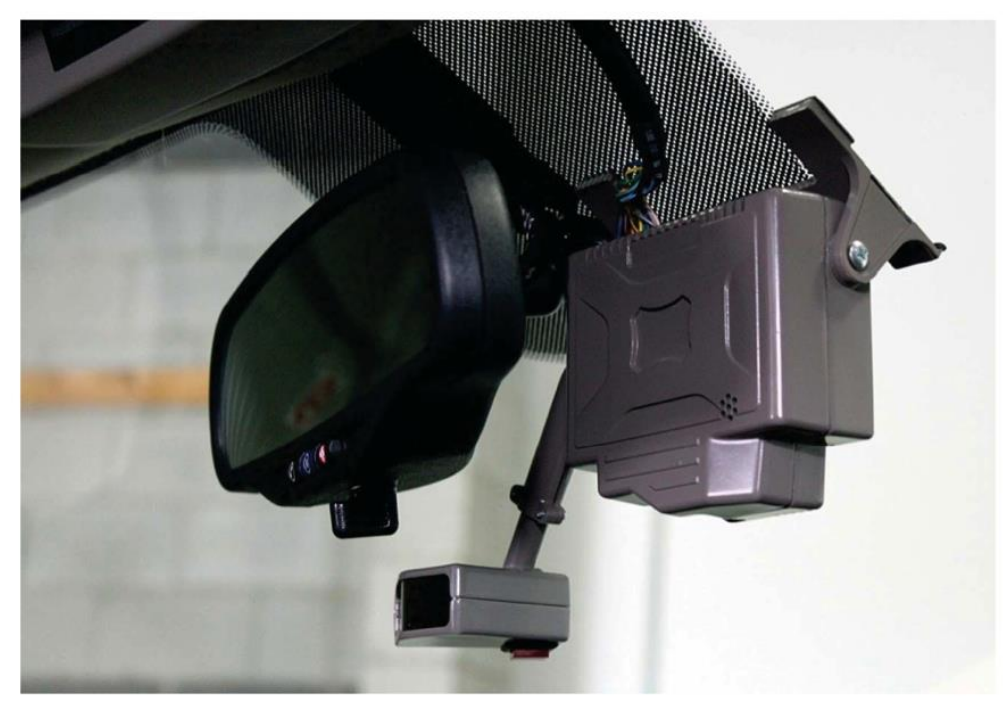

Figure 1. SHRP-2 camera unit mounted behind rearview mirror [CAMPBELL]

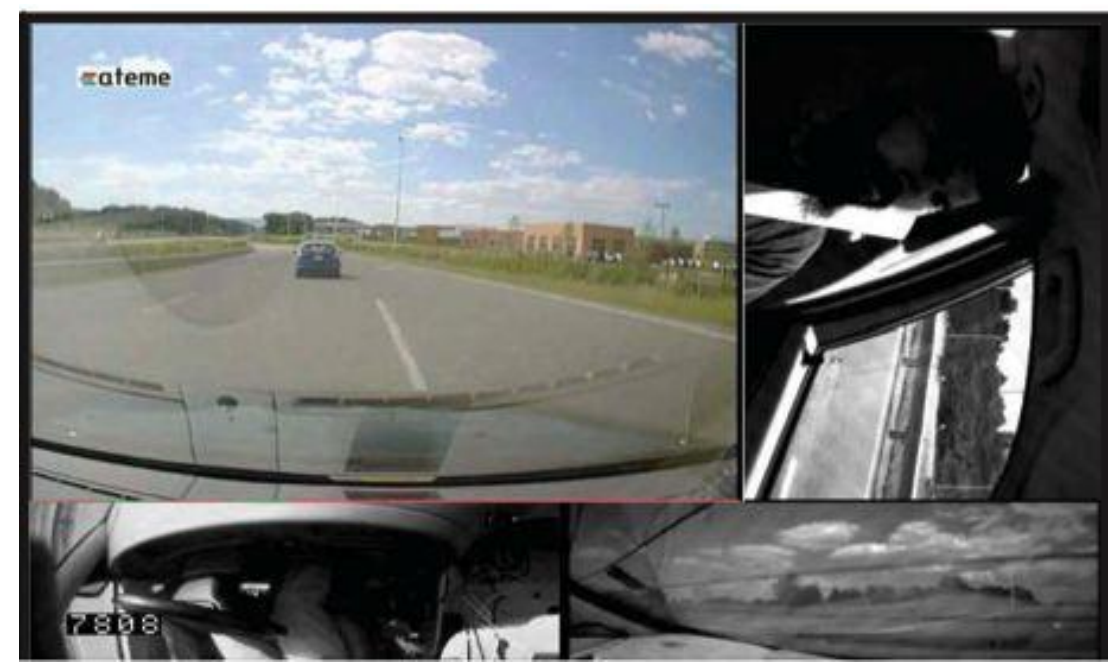

Figure 2. SHRP-2 composite view as recorded by DAS [CAMPBELL]

For most of the tests, the vehicle was parked under a tarp to protect the participants from sun and the tarp was periodically adjusted to reduce the impact of bright sunlight in the cab of the car. 


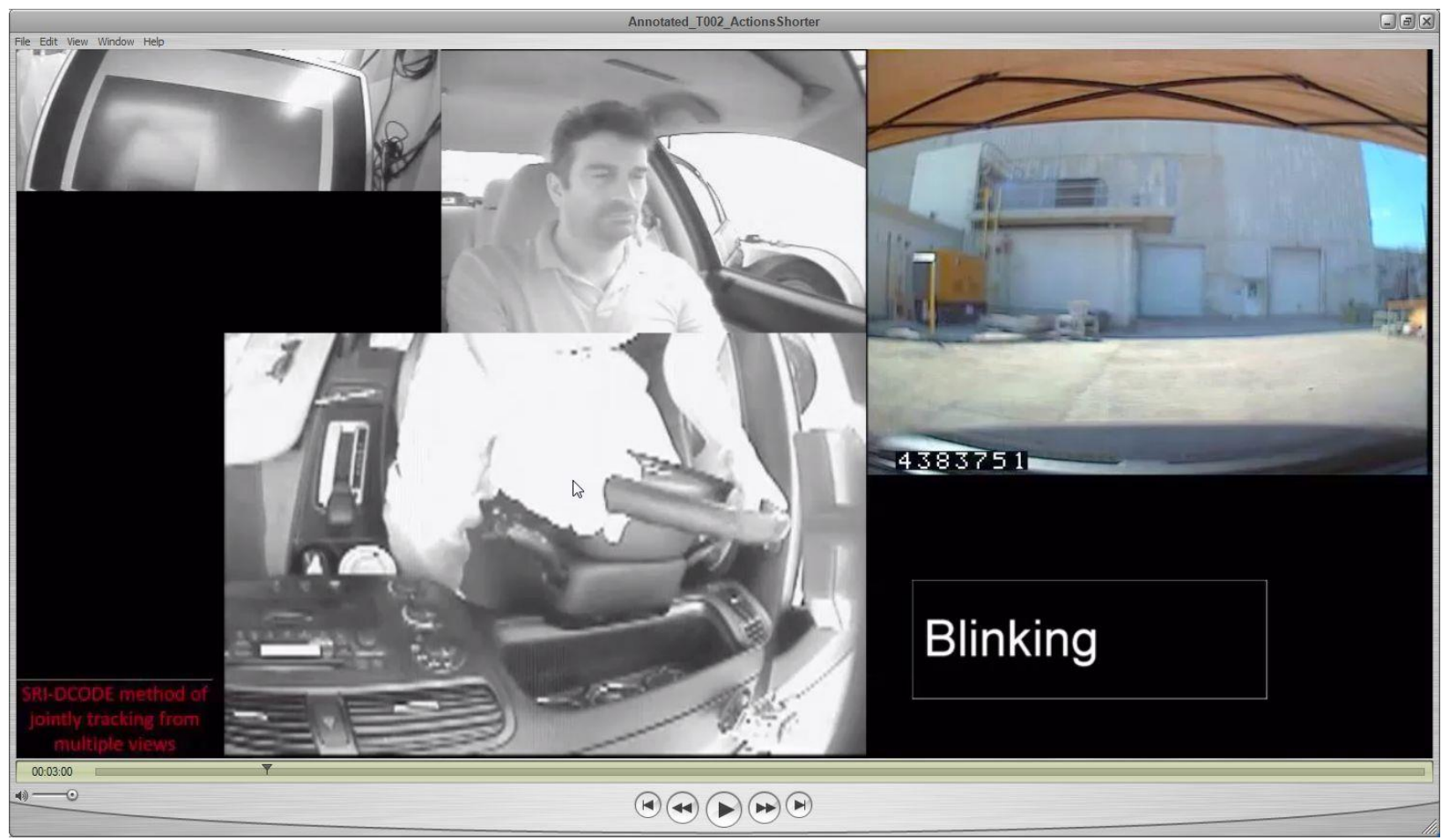

Figure 3. Annotated video view.

\section{STUDY DESIGN}

With the exception of a brief driving study, all of the participant actions were performed in a parked car. For the longer experiments-HeadPose, Check, ActionsShorter, and GlassAndCell - the participants responded to recorded audio instructions. For the HeadPose and Check experiments, the instructions ran through a sequence of steps five times. For the other experiments, the instructions were completed three times for a series of actions. After conducting experiments with participants T002 and T003, the verbal recordings were amended to run slightly quicker, so the sequence and relative timing of those participants' actions might be slightly different from later trials, but this is reflected in the annotated data.

For each experiment, the following files were generated.

- Face video file - in proper orientation

- Rear video file

- Dash video file (like the hands file for VTTI data sets)

- Front video

- Combined video (SRI-DCODE method of dual hand-face tracking)

- Annotated video (combined video with addition of text showing current annotated action) 
- A cropped video file that represents data from the GoPro camera situated directly in front of the driver

- An AlignedGoProDas video file that shows the combined camera views, annotations, and the cropped video from the GoPro camera superimposed on the part of the front image. A csv file showing the corresponding GoPro and DAS frames used for synchronizing this video is included. (It was impractical to modify circuitry to trigger the GoPro and DAS to start at the same time.)

The videos were later reviewed to ensure that the experiments' scripted actions were correctly executed and completed and that a consistent start and stop time for each annotated action was recorded. In particular, for HeadPose, labeled actions such as "P Side Window" were annotated from the time the participant begins to move to complete the action, through actually viewing the described locations or action and then returning to looking straight ahead and simulate driving. If the participant "missed" or got confused about the action to execute, a "-1" was set as the start and stop frame for the action.

\section{ANNOTATIONS}

The annotations provided with the data set are stored in a MATLAB .MAT format and in a csv format for each table within the .MAT file. The following is a list of tables and the type of information within:

\begin{tabular}{ll}
\hline \multicolumn{1}{c}{ TableName } & \multicolumn{1}{c}{ Description of Contents } \\
ORNLTrialTable & $\begin{array}{l}\text { Lists participant identifiers, their gender, whether they typically wear glasses, and } \\
\text { references to high resolution still photos of the participants. } \\
\text { ORNLFilesTable }\end{array}$ \\
Enumerates the video files included in the release, which video category or experiment \\
the file relates to, and whether the video is from the Dash, Face, Front, or Rear camera \\
or is a composite view. Also includes the number of frames in the video and the frame \\
rate. \\
Enumerates the participants, the video category and video source, the start and end DAS \\
frame of the description (described action), and the INQSCRIBE time stamp values.
\end{tabular}

\section{AUTOGENERATED FACIAL ANNOTATIONS}

Accurate location of the face or significant movements of its location can be important indicators of a driver's distraction or actions. To help provide some additional information of potential interest to researchers, an automated face detector was run on all the Face videos and all the GoPro videos. The

${ }^{1}$ INQSCRIBE is a video-annotation tool used to perform the annotation. 
results of RetinaFace [DENG] presently one of the most accurate state of the art face detectors, is published on this dataset to be of potential use to researchers.

The annotations provided are in the form of a csv file for each video with each line of the file indicating a face detection and its landmarks. There is a field for the frame, which detected face (not tracked consistently - the detect_id $=0$ face in one frame may be the detect_id=1 face in another), the confidence score for the detection, the bounding box details, and 5 landmarks corresponding to the eyes, tip of nose, and the corners of the mouth. These annotations are not validated, but those with confidence scores of .95 and greater generally seem to be pretty accurate with this data. Confidence scores below .9 may often be false detections for this dataset.

\section{ACKNOWLEDGEMENTS}

We acknowledge the support of the Exploratory Advanced Research Program of the Federal Highway Administration. We also acknowledge the assistance of Virginia Tech Transportation Institute.

\section{REFERENCES}

[HANKEY] Hankey, Jonathan M., Miguel A. Perez, and Julie A. McClafferty. Description of the SHRP 2 naturalistic database and the crash, near-crash, and baseline data sets. Virginia Tech Transportation Institute, 2016.

[VNAV] VectorNav VN100 https://www.vectornav.com/products/VN-100

[CAMPBELL] Campbell, Kenneth L. "The SHRP 2 naturalistic driving study: Addressing driver performance and behavior in traffic safety." Tr News 282 (2012).

[DENG] Deng, Jiankang \& Guo, Jia \& Zhou, Yuxiang \& Yu, Jinke \& Kotsia, Irene \& Zafeiriou, Stefanos. 2019. RetinaFace: Single-stage Dense Face Localisation in the Wild. https://arxiv.org/abs/1905.00641

[INSIGHT] Virginia Tech Transportation Institute. 2018. Insight Data Access Website. https://insight.shrp2nds.us/. 